\title{
MIXED PLAIN BOUNDARY VALUE PROBLEM OF ELASTICITY FOR A RECTANGULAR DOMAIN
}

\author{
D. Nerukh*, O. Pozhylenkov ${ }^{* *}$, N. Vaysfeld ${ }^{* *}$
}

\begin{abstract}
Mixed plain boundary value problem is considered for the elastic rectangular domain. The conditions of first main elasticity problem are given at the lateral sides; the conditions of smooth contact are given at the base. The rectangular domain is under impact of the exterior static load at its upper edge. With the integral transformation method the problem is reduced to a vector boundary problem, where the unknown vector consists of the displacement's transforms. The solution of vector boundary problem is constructed with the help of matrix differential calculations and has the unknown function. To find this function the integral singular equation is derived and solved with the help of the orthogonal polynomial method. The stress state of the rectangular domain is investigated depending on its size and kinds of impact.
\end{abstract}

Keywords: Mixed problem, Elasticity, Rectangular domain, Singular integral equation, Static load.

\section{Introduction}

The problem of a rectangular domain stress estimation is not a new one, nevertheless a lot of unsolved issues remain. This problem was considered and solved in the different statements important to the engineering applications as with the help analytical methods so and with numerical ones. To the last direction one can reference the papers, where the boundary element-free method (BEFM) was applied to two dimensional problems of elasticity. This method is a direct numerical method which combines the boundary integral equation method and an improved moving least-square approximation. This method, as it was stated at (Liew K. M., 2005), gives the higher computational accuracy. Another popular solving methods are well known finite element methods. For example, at (Oden J. T., 1982) the discussion of the condition's type necessary for the penalty methods to provide a basis for the stable and convergent finite element schemes is proposed. Many benefits of the numerical methods can be attributed by their existence at many numerical software applications, easy for using by the engineers.

But if one need to provide the calculation of the stress at the rectangular domain in the neighborhood of the angular points, the numerical methods lose their efficiency as it is known. These points of the boundary condition changing cause the stress with a special order of a singularity. To take these singularities at the attention, to propose the method which solve a problem for a rectangular domain with regard of such singularities existence, one must use the analytical approaches (Shyam N., 1983).

The well known paper (Vihak V. M., 1998) was one of the pioneer papers in this direction. The solution of the plane thermoelasticity problem for a rectangular domain was constructed with the help of new solving method. This method permits the construction of an analytical solution, corresponding to SaintVenan principle in the form of trigonometric series expansion using a complete orthogonal set of the

\footnotetext{
* Dmitriy Nerukh, DSc.: Aston University, Aston Triangle,; B4 7ET, Birmigham, United Kingdom. D.Nerukh@aston.ac.uk

** Oleksii Pozhylenkov, Master of Applied mathematics: Odesa Mechnikov University, Dvoryanskaya str. 2,; 65082, Odesa; Ukraine, leshiy12345678@gmail.com

** Natalya Vaysfeld, DSc., Prof.: Odesa Mechnikov University, Dvoryanskaya str. 2,; 65082, Odesa; Ukraine, vaysfeld@onu.edu.ua
} 
eigenfunctions and associated functions. These investigations were successfully continued by (Tokovyy, 2002).

Another analytical method of the plane two dimensional problem solving for a rectangular domain was proposed at the papers of Prof. G. Popov (Popov G., 1982). At the paper (Popov G., 2014) the plane mixed boundary value problem of elasticity on a rectangular domain is solved exactly. With the help of Fourier transformation the one-dimensional vector boundary problem in the transformation`s domain is obtained. The components of the unknown vector are the displacement transformations. The problem is solved exactly with the methods of the matrix differential calculations. The constructed vector is inversed by the corresponding formulas of inverse Fourier transformation, so the displacements expressions are found in the form of Fourier series. The numerical investigation of the stress in dependence of the external loading value and domain`s size is presented.

The novelty of the presented paper is in the application of the new approach (Popov G., 2011) to the solving of the first main elasticity problem for a rectangular domain. This new method of solution`s deriving reduces the stated problem to a singular integral equation which is solved with the orthogonal polynomial method taking into consideration the real singularities of the solution at the ends of the integration interval. The stress state of a domain was investigated depending on a load properties and domain size.

\section{The statement of problem.}

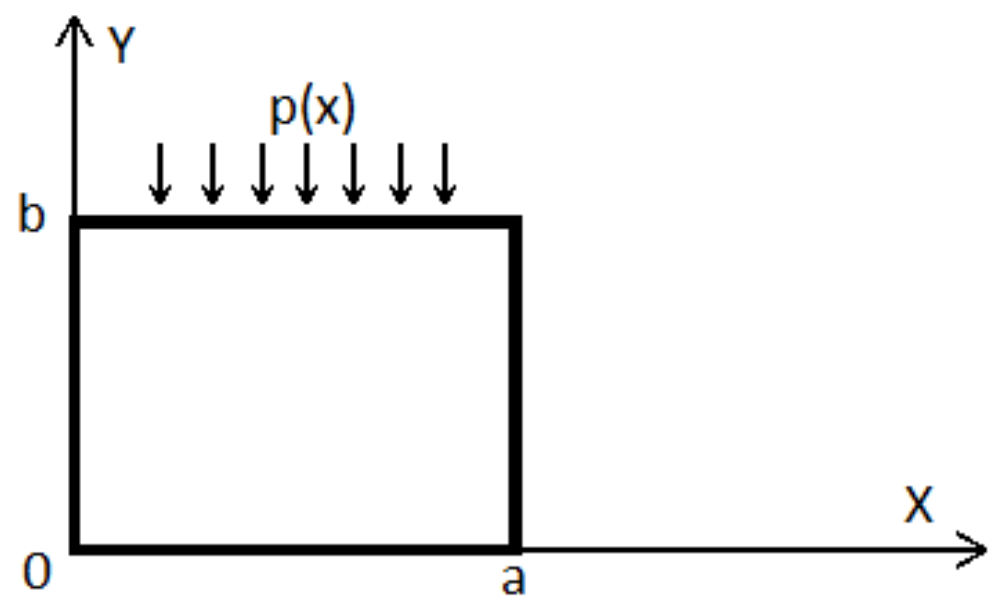

Fig. 1 Geometry of the problem

The elastic rectangular domain $0<x<a, 0<y<b$ coefficient) meets a load at the upper face of the domain

$$
\sigma_{y}(x, b)=-p(x), \tau_{y x}(x, b)=0
$$

The lower base at the conditions ideal contact

$$
u_{y}(x, 0)=0, \tau_{x y}(x, 0)=0
$$

The conditions of the main elasticity problem are fulfilled at the lateral sides

$$
\begin{aligned}
& \sigma_{x}(0, y)=0, \tau_{x y}(0, y)=0,0<y<b \\
& \sigma_{x}(a, y)=0, \tau_{x y}(a, y)=0,0<y<b
\end{aligned}
$$

It is required to estimate the stress state of the rectangular domain (1) satisfying the boundary conditions (2) - (4) and the equilibrium equations (Nowacki, 1970)

$$
\begin{aligned}
& U^{\prime \prime}(x, y)+U^{* *}(x, y)+\mu_{0}\left(U^{\prime \prime}(x, y)+V^{\prime *}(x, y)\right)=0 \\
& V^{\prime \prime}(x, y)+V^{* *}(x, y)+\mu_{0}\left(V^{* *}(x, y)+U^{\prime *}(x, y)\right)=0
\end{aligned}
$$


Here $U(x, y)=u_{x}(x, y), V(x, y)=u_{y}(x, y), f^{\prime}(x, y)=\frac{\partial f(x, y)}{\partial x}, f^{*}(x, y)=\frac{\partial f(x, y)}{\partial y}, \mu_{0}=\frac{1}{1-2 \mu}$.

\section{The problem solving.}

The Fourier`s transforms are applied to the equations (5) with the scheme

$$
\left(\begin{array}{l}
U_{n}(y) \\
V_{n}(y)
\end{array}\right)=\int_{0}^{a}\left(\begin{array}{l}
U(x, y) * \sin \left(\alpha_{n} x\right) \\
V(x, y) * \cos \left(\alpha_{n} x\right)
\end{array}\right) d x, \alpha_{n}=\frac{\pi n}{a}
$$

It leads to the inhomogeneous system of the ordinary differential equations in the transform`s domain

$$
\begin{gathered}
U_{n}^{\prime \prime}(y)-\alpha_{n}^{2}\left(1+\mu_{0}\right) U_{n}(y)-\mu_{0} \alpha_{n} V_{n}^{\prime}(y)=f_{n}^{1}(y), \\
\left(1+\mu_{0}\right) V_{n}^{\prime \prime}(y)-\alpha_{n}^{2} V_{n}(y)+\mu_{0} \alpha_{n}\left(1+\mu_{0}\right) U_{n}^{\prime}(y)=f_{n}^{2}(y)
\end{gathered}
$$

here $f_{n}^{1}(y)=\left(1+\mu_{0}\right) X_{n}(y), f_{n}^{2}(y)=\mu_{0} X_{n}^{\prime}(y)$

Boundary conditions (2), (3) are reformulated in the terms of the displacements.

$$
\begin{gathered}
U_{n}^{\prime}(0)=0, V_{n}(0)=0 \\
\alpha_{n} V_{n}(b)-U_{n}^{\prime}(b)=0, \alpha_{n} \mu U_{n}(b)-(1-\mu) V_{n}^{\prime}(b)=p_{n}
\end{gathered}
$$

To formulate the vector boundary state problem the vector and matrix are introduced

$$
\begin{gathered}
I=\left(\begin{array}{cc}
1 & 0 \\
0 & 1+\mu_{0}
\end{array}\right), P=\left(\begin{array}{cc}
0 & -\mu_{0} \alpha_{n} \\
0 & 1+\mu_{0}
\end{array}\right) \\
Q=\left(\begin{array}{cc}
-\mu_{0} \alpha_{n}^{2}\left(1+\mu_{0}\right) & 0 \\
0 & \alpha_{n}^{2}
\end{array}\right), Z_{n}(y)=\left(\begin{array}{l}
U_{n}(y) \\
V_{n}(y)
\end{array}\right) \\
A_{1}=\left(\begin{array}{ll}
1 & 0 \\
0 & 0
\end{array}\right), B_{1}=\left(\begin{array}{ll}
0 & 0 \\
0 & 1
\end{array}\right), f_{n}(y)=\left(\begin{array}{l}
f_{1}(y) \\
f_{2}(y)
\end{array}\right) \\
A_{2}=\left(\begin{array}{cc}
-1 & 0 \\
0 & \mu-1
\end{array}\right), B_{2}=\left(\begin{array}{cc}
0 & \alpha_{n} \\
\alpha_{n} \mu & 0
\end{array}\right)
\end{gathered}
$$

With the help of the introduced matrixes, the differential operator of second order is constructed

$$
L_{2}\left(Z_{n}(y)\right)=I Z_{n}^{\prime \prime}(y)+P Z_{n}^{\prime}(y)+Q Z_{n}(y)
$$

The vector boundary in the transform`s domain is formulated with the help of the introduced operator (9)

$$
\begin{gathered}
L_{2}\left(Z_{n}(y)\right)=f_{n}(y) \\
U_{i}\left(Z_{n}(y)\right)=0, i=1,2
\end{gathered}
$$

here $U_{i}\left(Z_{n}(y)\right)=A_{i} Z_{n}^{\prime}\left(b_{i}\right)+B_{i} Z_{n}\left(b_{i}\right), b_{1}=0, b_{2}=b$

To solve this halfhomogeneous vector boundary problem the Green`s matrix-function is constructed. The matrix differential calculations were used to constructed it. The matrix equation $L_{2} Y(y)=f(y)$ was considered first and solution of the corresponded homogeneous matrix equation was constructed. Matrix $Y(y)$ was chosen in the form $Y(y)=e^{\xi y} E$ ( $E$ is an unit matrix) and substituted into the equation. The equality $L_{2} e^{\xi y} I=M(\xi) e^{\xi y}$ is derived and solution is found with help of formula $Y(y)=\frac{1}{2 \pi i} \oint_{C} e^{\xi y} M^{-1}(\xi) d \xi$ (Gantmakher, 1998). The calculation of the integral requires to know all poles of the under integral function. To do it the determinant of the matrix $M(\xi)$ was found $\operatorname{det} M(\xi)=$ $\left(\xi^{2}-\alpha_{n}^{2}\right)^{2}$. After contour integration procedure the two linear independent solutions of the matrix equation were found.

$$
\begin{aligned}
& Y_{0 n}(y)=\frac{e^{\alpha_{n} y}}{4\left(1+\mu_{0}\right)}\left(\begin{array}{cc}
\mu_{0} \alpha_{n} y+\mu_{0}+2 & \mu_{0} y \\
-\mu_{0} y & \frac{-\mu_{0} \alpha_{n} y-\mu_{0}-2}{\alpha_{n}}
\end{array}\right) \\
& Y_{1 n}(y)=\frac{e^{-\alpha_{n} y}}{4\left(1+\mu_{0}\right)}\left(\begin{array}{cc}
\mu_{0} \alpha_{n} y-\mu_{0}-2 & -\mu_{0} y \\
-\mu_{0} y & \frac{-\mu_{0} \alpha_{n} y+\mu_{0}+2}{\alpha_{n}}
\end{array}\right)
\end{aligned}
$$


The solution of homogeneous vector equation was derived

$$
Z_{n}(y)=Y_{0 n}(y)\left(\begin{array}{l}
C_{1} \\
C_{2}
\end{array}\right)+Y_{1 n}\left(\begin{array}{l}
C_{3} \\
C_{4}
\end{array}\right)
$$

The Green`s matrix-function construction in the linear expansion $G(y, \xi)=\frac{2}{b} \sum_{n=0}^{\infty}{ }^{\prime} H\left(y, \alpha_{n}\right) \Omega^{-1}\left(\alpha_{n}\right) H\left(\xi, \alpha_{n}\right)$ (a stroke means that zeroth member is multiplied by $1 / 2$ ) is finishing the deriving of the inhomogeneous problem (10) solution. The final formula in the transform`s domain has the form

$$
Z_{n}(y)=Y_{0 n}(y)\left(\begin{array}{l}
C_{1} \\
C_{2}
\end{array}\right)+Y_{1 n}\left(\begin{array}{l}
C_{3} \\
C_{4}
\end{array}\right)+\int_{0}^{b} G(y, \xi) f_{n}(\xi) d \xi,
$$

where constants $C_{i}, i=\overline{1,4}$ are found from the boundary conditions. The application of the inverse Furier`s formula would finalize the stated problem`s solution if the unknown function $X_{n}(y)$ is known.

To find this unknown function the condition (2) should be satisfied. It leads to the singular integral equation with regard to unknown function $X_{n}(y)$. This equation was solved with the help of the orthogonal polynomial's method and was reduced to the infinite system of linear algebraic equations.

The stretching stress appearing at the bottom face of the rectangular domain was investigated. The conditions of its existence depending on geometrical parameters of the domain and properties of a load were stated.

\section{Concluding remarks.}

The proposed method was applied to solve the first main elasticity problem for a rectangular domain. It reduces the problem to the singular integral equation. The orthogonal polynomial method was used to derive the solution. The conditions of stretching stress existence were investigated depending on domains size and loads properties.

The future development is connected with nonstationary elasticity problem for a rectangular domain where the proposed solving method will be applied.

\section{Acknowledgement}

The research is supported by Erasmus+ exchange mobility program between Aston University and Odesa Mechnikova University.

\section{References}

Liew K. M., Yuming Cheng, Kitipornchai S. (2005) Boundary element-free method (BEFM) and application to twodimensional elasticity problems. International journal for Numerical Methods in Engineering. Volume 65, Issue 8.

Oden J. T., Kikuchi N. (1982) Finite element methods for constrained problems in elasticity. International journal for Numerical Methods in Engineering. Volume 18, Issue 5.

Shyam N. Prasad, Sailendra N. Chatterjee. (1973) Some mixed boundary value problems of elasticity in a rectangular domain. International Journal of Solids and Structures. Volume 9, Issue 10, pages 1193-1210.

Vihak V. M., Yuzvyak M. Y., Yasinskij A. V. (1998) The solution of the plane thermoelasticity problem for a rectangular domain. Journal of Thermal Stresses. Volume 21, Issue 5.

Vihak V. M., Tokovyy Yu. (2002) Construction elementary solutions of the elasticity plane problem for the rectangular domain. International applied mechanics. Volume 38, Issue 7, pages 79-87.

Popov G., (1982) The elastic stress concentration around dies, cuts, thin inclusions and reinforcements (in Russian), Nauka, Moscow.

Popov G., Vaysfeld N., Zozulevich B. (2014) The exact solution of elasticity mixed plain boundary value problem in a rectangular domain. 20-th International Conference Engineering Mechanics. Srvatka, Czech Republic.

Popov G., Vaysfeld N. (2011) The steady-one oscillations of the elastic infinite come loaded at a vertex by a concentrated force. Acta Mechanica. 221, Issue 3-4, pp. 261-270.

Nowacki W. (1970) The theory of elasticity. Panstwowe Wydawnictwo Naukowe, Warszawa, (in Polish).

Gantmakher F. R. (1998) The theory of matrices. AMS Chelsea Publishing, Providence, Rohde Island. 